\title{
Corela
}

Cognition, représentation, langage

7-1 2009

Vol. $7, \mathrm{n}^{\circ} 1$

\section{Informer sans s'engager : modélisation de la dynamique énonciative dans les sujets d'actualité}

\author{
Marie Chagnoux
}

\section{OpenEdition}

\section{Journals}

Édition électronique

URL : http://journals.openedition.org/corela/162

DOI : $10.4000 /$ corela. 162

ISSN : 1638-573X

Éditeur

Cercle linguistique du Centre et de I'Ouest - CerLICO

Référence électronique

Marie Chagnoux, «Informer sans s'engager : modélisation de la dynamique énonciative dans les sujets d'actualité », Corela [En ligne], 7-1 | 2009, mis en ligne le 26 juin 2009, consulté le 19 avril 2019. URL : http://journals.openedition.org/corela/162 ; DOI : 10.4000/corela.162

Ce document a été généré automatiquement le 19 avril 2019

\section{(c) (1) (2)}

Corela - cognition, représentation, langage est mis à disposition selon les termes de la licence Creative Commons Attribution - Pas d'Utilisation Commerciale - Partage dans les Mêmes Conditions 4.0 International. 


\title{
Informer sans s'engager : modélisation de la dynamique énonciative dans les sujets d'actualité
}

\author{
Marie Chagnoux
}

\section{Informer sans s'engager}

1 La révolution médiatique opérée par Internet a renforcé ces dernières années un certain nombre de phénomènes qui modifient sensiblement le caractère supposé objectif de l'information délivrée en ligne par opposition aux supports plus traditionnels. À titre d'exemples, citons trois phénomènes distincts qui illustrent cette évolution. Le premier est corrélé à l'essor des sites collaboratifs. L'encyclopédie Wikipedia ${ }^{1}$ propose des articles écrits à plusieurs mains : l'information délivrée n'est alors plus le point de vue d'un auteur sur un objet mais la synthèse d'une pluralité de points de vue. Le second réside dans l'extrême rapidité avec laquelle une information est disponible. La mise en ligne immédiate des données de l'AFP ne permet pas toujours d'en vérifier la véracité et il revient alors au journaliste de préciser le caractère incertain de l'information qu'il est en train de relater. Ainsi dans les heures qui suivent un accident d'avion, le bilan des morts et des blessés est toujours donné au conditionnel et ce n'est que plus tard que le bilan définitif sera exprimé au passé composé comme le montrent les extraits [1] et [2] tirés du site de l'Express'2.

[1] L'accident d'avion à Phuket aurait fait 61 morts et 40 disparus. ${ }^{3}$

[2] L'accident d'un avion de ligne thaillandais avec 130 personnes à bord, dimanche

sur l'aéroport de Phuket (sud), a fait 89 morts, selon un dernier bilan. ${ }^{4}$

Le troisième est lié à l'internationalisation du support : l'information délivrée peut être lue par tous et partout dans le monde. Elle est donc accessible à des communautés de culture, de sensibilité et d'opinions différentes et le choix du lexique trahit souvent l'appartenance de l'auteur à une communauté : le même individu peut à la fois être vu comme un terroriste en Russie et comme un résistant en Tchétchénie. Ainsi, à sa mort, 
Chamil Bassaev fut qualifié de "terroriste numéro un » de la Russie ${ }^{5}$ par un quotidien français tandis qu'un autre le désignait plus honorablement comme le « chef de guerre tchétchène $»^{6}$. Tous ces facteurs incitent de plus en plus les auteurs d'articles en ligne à déléguer la responsabilité d'une information à un tiers ou à insister sur le caractère plausible ou incertain de l'information donnée.

tes journalistes à s'engager sur la véracité des informations qu'ils délivrent a été particulièrement perceptible durant l'été 2006 autour du conflit armé israélopalestinien où des sources d'information contradictoires s'affrontaient. Nous avons à cette époque collecté un corpus varié contenant à la fois des textes pro-israéliens, des textes pro-libanais et des textes visant a priori à l'objectivité. D'une part, l'intérêt de ce corpus était de montrer comment un même événement, la capture de deux soldats israéliens, pouvait être narré par deux communautés antagonistes, en l'occurrence la communauté pro-israélienne et la communauté pro-libanaise. D'autre part, il s'agissait d'analyser comment les médias arrivaient à concilier les deux points de vue sans prendre parti, en articulant plusieurs référentiels. Ce corpus d'une vingtaine de textes nous a permis de mettre en oeuvre le cadre méthodologique général afin de montrer qu'analyser la structure discursive fait émerger des éléments de nature typologique qui permettent de repérer les sujets d'actualité polémiques.

Pour présenter ces travaux, nous exposerons dans un premier temps les principes théoriques de ce cadre méthodologique. Dans un second temps, nous montrerons comment ce cadre est mis en oeuvre pour associer une représentation graphique à la structure discursive. Enfin, la dernière partie présentera l'exploitation des arbres construits.

\section{Repérer et modéliser les variations de prise en charge énonciatives}

\subsection{Les variations de prise en charge énonciative}

5 Comme le définit [Culioli 1980 :184], le concept de prise en charge repose sur le fait de « dire ce qu'on croit (être vrai) ». Ainsi, tout énoncé suppose la prise en charge implicite des propos. Dans les exemples [3], en énonçant des faits, les énonciateurs assurent implicitement de la véracité de leurs propos.

[3a] Au sud, à Rafah, le point de passage est toujours fermé par l'armée d'occupation, une femme et un enfant y sont morts hier.'

[3b] 8 Israéliens ont été tués jeudi 3 août, dans l'après-midi, suite à une salve de roquettes tirées par le Hezbollah sur la Galilée occidentale. ${ }^{8}$

Cependant, D. Maingueneau précise que certains faits de langues « exigent que l'on sépare l'instance qui énonce et celle qui assume la responsabilité du dire $»^{9}$. Cette dissociation entre «celui qui parle » et «celui qui prend en charge ce qui est dit» est opérée par l'énonciateur ${ }^{10}$ :

Le discours n'est discours que s'il est rapporté à une instance qui à la fois se pose comme source des repérages personnels, temporels, spatiaux et indique quelle attitude il adopte à l'égard de ce qui est dit et de son interlocuteur (processus de modalisation). Le locuteur peut moduler son degré d'adhésion («Peut-être qu'il pleut »), en attribuer la responsabilité à quelqu'un d'autre («Selon Paul il pleut ») $[\ldots]^{11}$ 
7 De l'effacement énonciatif étudié par [Rabatel 2006] aux variations journalistiques proposées par [Rosier 1993], il existe une gradualité du degré de prise en charge énonciatif. Les extraits du corpus des exemples [4] illustrent la diversité des mécanismes langagiers par laquelle un énonciateur peut exposer des faits ou des opinions sans en assumer complètement la prise en charge.

[4a] Selon le ministre canadien, il y aurait huit ressortissants canadiens parmi les victimes.

[4b] Selon un général, des commandos terrestres sont entrés sur le territoire libanais.

[4c] Le 30 juillet, Human Rights Watch considère qu'Israël a commis des crimes de guerre et le juge responsable des victimes du bombardement de Cana[...]

[4d] « Les deux prisonniers israéliens ont été transférés dans un lieu sûr ", a déclaré le Hezbollah.

[4e] La police libanaise affirme que l'aviation israélienne a entièrement détruit un lieu de prière et de rassemblement chiite à Budaï, près de Baalbeck, dans l'est du Liban.

[4f] Le 31 juillet, un patrouilleur israélien Sa'ar 4 aurait été atteint au large de Tyr par un missile inconnu du Hezbollas, bien que l'armée israélienne ait démenti l'information.

[4g] Cela pourrait également ajouter des dégâts terrestres à la catastrophe écologique.

Nous retenons ici deux mécanismes: le premier consiste à attribuer des propos à une autre source énonciative par le biais de citations, le second «module le degré d'adhésion » aux propos par le biais de modalisation.

\subsubsection{Les mécanismes citationnels}

9 Pour marquer sa distance avec les situations exprimées, l'énonciateur peut donc attribuer la responsabilité de ce qui est dit à un tiers, phénomène qui instaure une rupture énonciative marquée. Ainsi, dans les exemples [4a-e], l'énonciateur convoque respectivement «le ministre canadien», « un général», «Human Rights Watch», «le Hezbollah» ou «la police libanaise». Dès lors, ce n'est pas le journaliste qui assume qu' «Israël a commis des crimes » mais un énonciateur second, l'organisme Human Rights Watch. Ces exemples montrent comment le recours à la citation permet de présenter des faits sans en assumer la portée.

Bien que certains auteurs préfèrent réserver le terme de citation au discours rapporté direct [Tuamarla 2000], nous l'utilisons comme le terme générique permettant de référer à tout discours rapportant les propos d'un tiers. Nous définissons donc la problématique de la citation à la suite de [Rosier 1999 : 125] comme la « mise en rapport de discours dont l'un créé un espace énonciatif particulier tandis que l'autre est mis à distance et attribué à une autre source de manière univoque ou non ».

11 Notre objectif n'est pas de caractériser les types de citation mais bien de repérer les segments attribués à cette autre source ${ }^{12}$ par rapport au reste du discours assumé directement par l'énonciateur. Ce repérage se base sur l'exploitation de marqueurs de surface qui indiquent cette mise à distance. Il peut s'agir d'indices typographiques comme les guillemets de [4d] ou de structures lexico-syntaxiques comme celles construites par les verbes introducteurs considérer, juger, déclarer, affirmer de [4c-e] ou la préposition Selon 


\subsubsection{Les mécanismes de modalisation}

12 Alors que les mécanismes citationnels portaient sur la "source énonciative ", les mécanismes de modalisation désignent «l'attitude du sujet parlant à l'égard de son propre énoncé » [Charaudeau et Maingueneau 2002 :382]. Parce que le terme de modalité recouvre des notions distinctes selon les domaines et les problématiques, nous restreignons ici les modalités au sens de [Le Querler $1996: 64]$ comme celles "par lesquelles le locuteur exprime son degré de certitude sur ce qu'il asserte $»^{13}$.

Les mécanismes de modalisation désignent alors ici tous les phénomènes par lesquels l'énonciateur va présenter un contenu propositionnel comme possible, probable, hypothétique, etc. afin de le distinguer du «être vrai» de la définition d'A. Culioli. Ici encore, le repérage des segments modalisés dans les exemples 4 s'appuient sur des marqueurs de surface comme le conditionnel comme dans [4a],[4f], [4g] ou le recours à des verbes modaux comme pouvoir dans [4g].

14 Comme pour les phénomènes citationnels, notre présentation des phénomènes de modalisation est très succincte car elle vise essentiellement à résumer des connaissances nécessaires pour comprendre le cadre méthodologique. Nous souhaitons montrer qu'à travers des phénomènes citationnels ou de modalisation, un énonciateur peut présenter des faits et des opinions en les prenant plus ou moins, voire même pas du tout, en charge. Dans tous les cas, on observe un mécanisme de rupture dans la prise en charge énonciative qui distingue le segment cité ou modalisé du reste de l'énoncé. Dans le cas de la citation, la rupture énonciative repose sur un changement d'énonciateur comme dans les exemples [3a-f] alors que dans le cas de la modalisation la rupture énonciative repose sur un changement de statut de l'énoncé. La comparaison des exemples [4a] et [4b] montre que les deux types de mécanismes peuvent s'associer : différents indices relevant de niveaux hiérarchiques distincts se croisent. Dans le premier exemple, la séquence selon + autorité citée introduite par un déterminant défini + conditionnel combine à la fois une source plus sûre que dans le second où l'autorité citée est introduite par un déterminant indéfini (un général) et la marque d'une plus grande distance (conditionnel versus passé composé). À nouveau, nous insistons sur le fait que les phénomènes linguistiques que nous citons ici relèvent de problématiques largement débattues et de traitements plus complexes, mais cette présentation succincte visait avant tout à rappeler rapidement au lecteur les principes sur lesquels se fonde l'analyse.

\subsection{Ruptures énonciatives et cohérence discursive}

15 Ainsi, tous les exemples de [4] ont en commun le fait de situer le propos sur un référentiel ${ }^{14}$ autre que celui de l'énonciateur, que ce soit sur le référentiel d'un tiers ou sur un référentiel modalisé de l'énonciateur lui-même. Les marqueurs de surface permettent au lecteur de passer sans ambiguïté d'un référentiel à l'autre en articulant les référentiels entre eux. L'exemple [5] témoigne de la possible coexistence dans un même texte de plusieurs référentiels de nature énonciative.

[5] À propos du lieu de l'enlèvement des deux soldats, les versions diffèrent. Les Israéliens indiquent qu'ils ont été capturés près de la ferme collective de Zarit en territoire israélien tout près de la frontière libanaise. De son côté, la police libanaise soutient que la capture s'est produite dans la région de Aïta al-Chaab en territoire libanais donc proche de la frontière libano-israélienne où une unité israélienne avait pénétré le matin même. ${ }^{15}$ 
On peut ici relever trois référentiels énonciatifs distincts :

- Le référentiel de l'énonciateur sur lequel sont validés trois faits - la divergence des versions de la capture et les deux déclarations antagonistes ;

- le référentiel des Israéliens introduit par «Les Israéliens indiquent qu'« et sur lequel est validé le fait que la capture a eu lieu près de la frontière libanaise (et donc en territoire israélien);

- le référentiel de la police libanaise introduit par «la police libanaise soutient que » et sur lequel est validé le fait que la capture a eu lieu en territoire libanais.

Seule la prise en compte de la structure discursive balisée par les marqueurs de surface permet de comprendre comment deux informations contradictoires peuvent figurer dans un même texte sans contrarier la cohérence de ce dernier.

Afin de rendre compte de la structure discursive, notre objectif est de modéliser les ruptures référentielles qui articulent entre eux différents segments textuels en repérant (i) les marqueurs de cohésion ou de rupture qui structurent le discours et (ii) les segments cohérents qui forment des blocs homogènes à l'intérieur d'un même référentiel. À partir de l'identification des segments et des articulations, le texte peut être considéré non comme une suite linéaire de propositions mais comme une organisation structurée et hiérarchisée de segments textuels.

\subsubsection{Identifier les ruptures de cohérence discursive}

D'autres cadres théoriques ont déjà abordé la question de la structuration de surface des textes. Le postulat commun est que la structure discursive s'élabore dans des espaces de validation qui s'ouvrent, se ferment et éventuellement s'emboîtent et à l'intérieur desquels le principe de cohérence est respecté. Une première approche vise à rechercher localement les segments sémantiquement cohérents. Ainsi [Charolles 1997] chercher à repérer des cadres de discours: ces cadres de discours «intègrent une ou plusieurs propositions en fonction de critères qui sont spécifiés par les expressions les introduisant $»^{16}$. Ces critères peuvent être thématiques [Porhiel 2001], temporels [Terran 2002], spatio-temporels [Charolles \& al. 2005], etc. Il est également possible de citer les travaux sur le médiatif menés par [Schrepfer 2006]. Comme les référentiels, les cadres de discours peuvent également être considérés comme des équivalents discursifs des espaces mentaux de [Fauconnier 1985]. Une autre approche consiste à représenter globalement la structure textuelle de manière hiérarchisée comme dans [Mann et Thompson 1988], [Hobbs 1990] [Cornish 2006] ou [Asher 1993].

Ces différentes perspectives ont pour objectif de dégager des blocs homogènes et/ou de rendre compte des éventuelles relations hiérarchiques entre ces blocs. L'originalité de notre approche par rapport à ces travaux est qu'elle vise à dépasser les notions de ruptures et de phénomènes locaux pour s'intéresser à la dynamicité qui permet de passer dans les textes d'un bloc à l'autre.

\subsubsection{Modéliser la structure discursive en reconstruisant la dynamique textuelle}

20 Nous proposons en effet de modéliser le cheminement de lecture entre les différents référentiels d'un texte. Notre représentation de la structure discursive se base sur la construction dynamique de l'organisation textuelle à partir de la lecture syntagmatique. Pour représenter et visualiser la structure discursive en référentiels, nous avons proposé dans [Chagnoux 2006] de modéliser la dynamique discursive sous forme de graphe. Cette 
représentation considère le texte comme un ensemble de segments en relation d'inclusion ou de succession. Elle permet de visualiser le cheminement qui s'opère entre les différents référentiels au fur et à mesure de la lecture syntagmatique en insistant sur les opérations de rupture repérées par les différents indices linguistiques. ${ }^{17}$

Cette méthodologie présente l'avantage (i) de pouvoir représenter les segments discursifs homogènes d'un texte ainsi que les relations hiérarchiques qu'ils entretiennent entre eux ; (ii) d'offrir une visualisation du parcours nécessairement dynamique de lecture ; (iii) de proposer un mécanisme de fermeture systématique des référentiels ouverts ; (iv) de pouvoir s'appliquer à tout texte, quel que soit son type ${ }^{18}$.

\section{Cadre méthodologique}

Dans cette partie, nous décrivons le cadre théorique utilisé, les éléments linguistiques sur lesquels il s'appuie ainsi que les principes méthodologiques de construction des graphes.

\subsection{Les référentiels énonciatifs et modaux}

La distinction de différents référentiels énonciatifs a été proposée par [Desclés 1980] pour rendre compte de distinctions aspectuelles entre des valeurs d'état, de processus et d'événements attribuées à des propositions. Notre analyse s'appuie ce modèle, mais s'en distingue car nous proposons une représentation alternative qui ignore les phénomènes aspectuels temporels fondamentaux pour rester à un niveau de surface et nous focaliser uniquement sur les mécanismes d'ouverture et de fermeture des référentiels énonciatifs. Nous présenterons donc la manière dont les textes articulent les différents référentiels sans considérer les intervalles de validation traditionnellement associés aux différentes propositions.

24 Parmi la typologie des référentiels proposée par [Desclés 1995] et précisée par [Chagnoux 2006], nous avons retenu trois types de référentiels pour rendre compte de la structure discursive ${ }^{19}$ :

- les référentiels énonciatifs (noté RE) ;

- les référentiels du possible (noté $\mathrm{RP}$ );

- les référentiels mentaux (noté RM).

\subsubsection{Les référentiels énonciatifs}

Les référentiels énonciatifs se définissent comme les référentiels à l'intérieur desquels le contenu est directement assumé par un énonciateur E. Tout texte suppose l'ouverture d'un référentiel énonciatif global, noté $\mathrm{RE}_{\mathrm{G}}$, dont les coordonnées sont celles de l'énonciateur principal. Par la suite, d'autres référentiels énonciatifs pourront s'ouvrir mais il s'agira de référentiels énonciatifs locaux, noté $\mathrm{RE}_{\mathrm{L}}$ dont les coordonnées sont liées à l'énonciateur du discours rapporté. Ainsi, l'exemple [5] articulait trois référentiels énonciatifs distincts :

- $\mathrm{RE}_{\mathrm{G}}$ : le référentiel de l'énonciateur;

- $\mathrm{RE}_{\mathrm{L}} 1$ : le référentiel des Israéliens ;

- $\mathrm{RE}_{\mathrm{L}} 2:$ le référentiel de la police libanaise. 
Par conséquent, quel que soit le mécanisme de construction, tous les discours rapportés, directs, indirects ou contextualisés convoquent un référentiel énonciatif local comme l'illustrent les exemples de [6] ${ }^{20}$ où les énoncés en gras appartiennent à REL.

[6a] Six villageois, dont trois enfants, ont été tués et six blessés dans le bombardement d'Aïtaroun, un village du Sud-Liban. A Aabba, près de Nabatiyé, à 70 $\mathrm{km}$ au sud-est de Beyrouth, des chasseurs bombardiers ont détruit plusieurs maisons. Selon le ministre canadien des Affaires étrangères, il y aurait huit ressortissants canadiens parmi les victimes.

[6b] «Toutes les victimes sont des employés de la compagnie nationale ferroviaire ", a précisé un pompier intervenant sur les lieux du drame.

[6c] Selon le président du Parlement libanais, Nabih Berri, «il existe une opportunité pour obtenir un cessez-le-feu » via une tierce partie.

[6c] A défaut d'un tel cessez-le-feu dans les 24 heures, «toute la région du ProcheOrient sera en danger ", a prévenu le président pro-syrien.

[6d] Peretz a en revanche réaffirmé que l'armée israélienne n'avait aucune intention de se « réinstaller» au Liban. "Nous n'avons aucune intention de nous enliser dans le bourbier libanais ", a-t-il ajouté.

\subsubsection{Les référentiels possibles}

Les référentiels possibles se définissent comme des référentiels à l'intérieur desquels le contenu est considéré comme éventuel, noté $\mathrm{RP}_{\mathrm{E}}$, ou contre-factuel, noté $\mathrm{RP}_{\mathrm{C}}$. Le référentiel possible éventuel marque la création d'un nouveau référentiel où les situations sont envisagées comme possibles ou probables, comme dans les exemples [7]

[7a] Le porte-parole de la diplomatie européenne, Javier Solana, est arrivé dimanche à Beyrouth. Il devrait s'entretenir avec le premier ministre libanais. ${ }^{21}$ [7b] Le 31 juillet, un patrouilleur israélien Sa'ar 4 aurait été atteint au large de Tyr par un missile inconnu du Hezbollah, bien que l'armée israélienne ait démenti l'information. ${ }^{22}$

Le référentiel possible contrefactuel se distingue du référentiel possible éventuel par la possibilité de confronter les situations qu'il décrit avec celles qui se sont déjà réalisées dans un autre référentiel comme dans l'exemple [8]

[8]Dan Gillerman, ambassadeur d'Israël à l'ONU, devant le Conseil de Sécurité (30 juillet) : «Ces personnes, y compris les femmes et les enfants, qui ont été tuées dans ce tragique accident, peuvent avoir été tuées par les tirs israéliens, mais ils sont victimes du Hezbollah. Ils sont victimes du terrorisme. S'il n'y avait pas de Hezbollah, ceci ne se serait jamais produit. $»^{23}$

Outre l'importance lexicale des verbes modaux (pouvoir, devoir, etc.) et de la construction syntaxique, il faut noter le rôle prépondérant des temps et des modes dans le repérage de ces référentiels. Ainsi, par exemple, parmi les possibles éventuels, il est possible de distinguer les possibles réels et les possibles potentiels. Ces distinctions sont marquées linguistiquement par l'emploi des temps verbaux. Si la proposition conditionnelle est au présent, le possible relève d'une projection de l'énonciateur. Si la proposition conditionnelle est à l'imparfait, le possible est plus strictement potentiel.

\subsubsection{Les référentiels mentaux}

Les référentiels mentaux se définissent comme des référentiels à l'intérieur desquels le contenu a un statut comparable à celui des méta-représentations décrites par [Recanati 2000] : il exprime un fait en même temps qu'une représentation de ce fait. Ainsi, ils sont le plus souvent convoqués par des propositions comme il pense que, il croit que, il imagine que, 
etc. Ils sont beaucoup moins importants dans notre corpus que les deux autres types de référentiels présentés plus haut.

\subsubsection{Exemples d'articulations entre référentiels} cohérents en terme de prise en charge énonciative. Ainsi, l'extrait $[9 \mathrm{a}]^{24}$ contient 3 segments qui sont identifiés en [9b] sur: REG un référentiel énonciatif global où $\mathrm{E}$ correspond à l'auteur de l'article, RPE un référentiel possible éventuel et REL un référentiel énonciatif local où $\mathrm{E}$ correspond au « général Amir Eshel ».

[9a]L'opération au Liban serait de longue durée. C'est ce que déclare le chef d'étatmajor adjoint de l'armée de l'air israélienne, le général Amir Eshel. Il prévient l'opinion internationale et intérieure que: «Il ne faut pas voir cela comme une question de jours. Cela va durer longtemps ».

[9b] $\mathrm{RE}_{\mathrm{G}}\left\{\mathrm{RP}_{\mathrm{E}}\right.$ \{L'opération au Liban serait de longue durée\}. C'est ce que déclare le chef d'état-major adjoint de l'armée de l'air israélienne, le général Amir Eshel. Il prévient l'opinion internationale et intérieure que : « $\mathrm{RE}_{\mathrm{L}}$ \{ Il ne faut pas voir cela comme une question de jours. Cela va durer longtemps\}».\}

\subsection{Cohésion et incohésion : les marqueurs linguistiques}

Comme nous l'avons indiqué en introduction, dans une perspective d'analyse de surface, l'ouverture et la fermeture des référentiels se traduisent linguistiquement par la présence de marqueurs linguistiques: des marqueurs strictement grammaticaux comme les temps verbaux dans les exemples [4f] et [4g] (« auraient été atteint », " pourrait »), des syntagmes adverbiaux ou prépositionnels comme dans les exemples [4a] et [4b] ("Selon le ministre», "Selon un général »), des marqueurs syntaxico-sémantiques ("considérer que », "affirmer que»,) comme dans les exemples [4c] et [4e] et des marqueurs typographiques comme dans $[4 \mathrm{~d}]$ (présence des guillemets).

Ces marqueurs linguistiques fonctionnent sur des principes de cohésion ou de rupture. Les marqueurs de cohésion assurent l'homogénéité discursive en liant ensemble les segments au sein d'un même bloc cohérent. Ainsi dans l'exemple [10], la conjonction de coordination « et » permet d'intégrer la troisième proposition dans le même segment que la seconde - soit le référentiel énonciatif local associé au Cheikh Nasrallah et non à celui associé à l'auteur de l'article.

[10]Comme l'indiquait le Cheikh Nasrallah, dirigeant du Hezbollah, la date de l'attaque de la patrouille israélienne n'avait pas été programmée à l'avance, et c'est un concours de circonstances favorables mais imprévues qui l'a provoqué

Il faut préciser que les marqueurs de cohésion sont des connecteurs, des adverbes, des temps verbaux concordant en système (dans le cas d'un système d'alternance d'imparfait et de passé simple par exemple) et qu'un même marqueur comme « et » peut être à la fois marqueur de cohésion comme dans l'exemple [10] et marqueur de rupture selon les éléments contextuels.

À l'inverse des marqueurs de cohésion, les marqueurs de rupture indiquent les transitions entre référentiels distincts. Il peut s'agir de marqueurs simples comme les indices typographiques tels que les guillemets, suivis ou non de deux points, ou d'ensembles de marqueurs comme dans l'exemple [11] qui mêle éléments lexicaux, syntaxiques et typographiques. 
[11] « Nos avions, nos chars et notre artillerie opèrent à l'intérieur du territoire libanais ", a déclaré un porte-parole militaire israélien, mercredi 12 juillet. ${ }^{25}$ constitue un indice: il ne s'agit donc pas simplement de repérer des marqueurs mais plutôt de repérer des configurations de marqueurs permettant de conclure ou non à l'intégration de segments dans un bloc. Nous utilisons ces marqueurs de surface comme des indices de la structure profonde du texte : c'est à partir de leur seule présence ou absence qu'est reconstruite la structure sous-jacente au discours.

\subsection{Les propriétés des graphes discursifs}

Ainsi, à partir de l'identification des marqueurs discursifs et des référentiels, il est possible de modéliser l'organisation textuelle sous forme de graphe ou d'arbre. Nous reviendrons plus loin sur la distinction entre graphe et arbre et présentons ici les principes de construction de la représentation graphique.

\subsubsection{Les nœuds}

Dans le graphe, les noeuds correspondent aux référentiels identifiés. Les graphes sont doublement étiquetés, chaque noeud possède une étiquette d'identification qui indique le type de référentiel qu'il dénote (énonciatif, possible, mental, ...) et une étiquette de contenue qui indique l'ensemble des propositions qui se rattachent à ce référentiel. Ainsi, par exemple $\mathrm{RE}_{\mathrm{L} 3}\left\{\mathrm{P}_{4}, \mathrm{P}_{5}\right\}$ désigne dans la figure 1 le cinquième référentiel du texte qui est le troisième énonciatif local et précise qu'il contient les propositions $\mathrm{P}_{4}$ et $\mathrm{P}_{5}$.

\section{Figure 1 : exemple de nœud.}

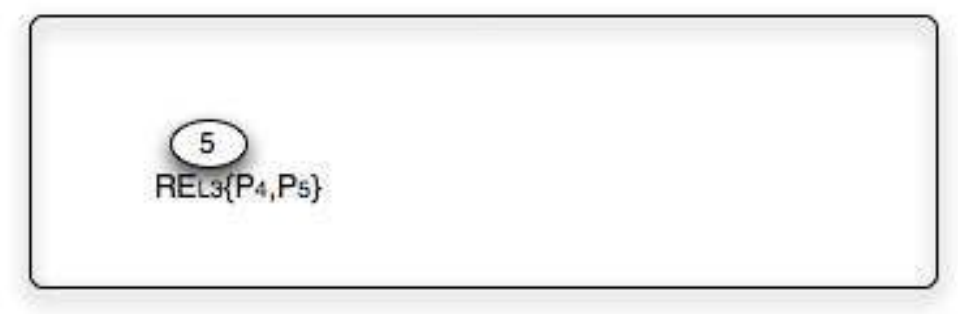

39 Le premier noeud du graphe occupe un statut particulier du point de vue théorique puisqu'il correspond toujours au référentiel énonciatif global. Même si aucun marqueur linguistique ne l'introduit explicitement, il est toujours présent et c'est à partir de ce premier noeud que se développe l'organisation textuelle. De plus, il est également le nœud terminal puisque le chemin de lecture s'achève à son niveau. Afin de permettre une identification rapide et aisée de ce nœud primordial, il est toujours représenté en noir.

\subsubsection{Les arcs}

Les arcs correspondent aux transitions entre référentiels et sont labellisés avec les marqueurs discursifs de rupture. Ils sont tous orientés et sont de deux types selon qu'ils portent une information de type linguistique ou non. Ils sont étiquetés par les marqueurs linguistiques indicateurs de rupture. Lorsqu'un arc ne porte pas ce type d'information, il 
indique un retour vers le référentiel immédiatement précédent et participe directement ou indirectement à la fermeture d'un ou plusieurs référentiels. Ainsi l'arc illustré par la figure 2 est un arc entre un le noeud $\mathrm{RE}_{\mathrm{L} 3}\left\{\mathrm{P}_{4}, \mathrm{P}_{5}\right\}$ et le noeud $\mathrm{RE}_{\mathrm{P}_{3}}\left\{\mathrm{P}_{6}\right\}$, l'arc est étiqueté avec le marqueur linguistique dont l'identification a permis de repérer la rupture, le verbe « devrait».

Figure 2 : exemple d'arc.

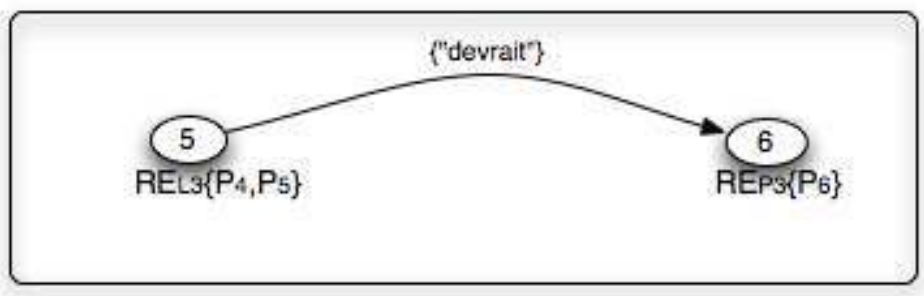

\subsubsection{Les règles de construction}

Un ensemble de règles définit les propriétés du graphe et en gouverne la construction. Elles peuvent se résumer comme suit :

- le graphe ne propose qu'un seul et même chemin qui commence et finit toujours sur $\mathrm{RE}_{\mathrm{G}}$;

- la prise en compte d'une nouvelle proposition répond à l'une des trois opérations suivantes : l'ouverture d'un nouveau référentiel, la fermeture du référentiel courant par un retour au niveau précédent ou la continuation du référentiel courant ;

- quel que soit le référentiel sur lequel se situe la dernière proposition du texte, il est nécessaire de revenir à $\mathrm{RE}_{\mathrm{G}}$ en fermant les uns après les autres tous les référentiels restés ouverts dans l'ordre dans lequel ils ont été ouverts. Ainsi, le point final d'un texte déclenchera la fermeture de tous les référentiels restés ouverts.

\section{Exemples de construction dynamique}

Pour illustrer l'analyse et la méthodologie mises en œuvre, nous proposons dans cette partie de les appliquer à deux extraits textuels: un extrait court pour lequel nous détaillons les principes de construction et un article complet, non détaillé, qui illustre le résultat obtenu sur un texte long.

\subsection{Application à un extrait court}

À partir des éléments précédemment définis, le tableau 1 présente, étape par étape, la construction du graphe associé à l'extrait de texte [12a] segmenté selon le format présenté dans l'exemple [12b]. Pour une meilleure lisibilité, des couleurs ont été attribuées aux nœuds, les nœuds des référentiels énonciatifs sont en violet et les nœuds des référentiels possibles sont en vert.

[12a] Depuis le début de la guerre, plus de 2070 roquettes sont tombées sur le nord d'Israël. Selon les estimations en Israël, la moitié des habitants de cette région, soit 800.000 personnes, l'ont fuie pour le centre et le sud du pays. Dans les villes qui ont été très gravement touchées, ce chiffre atteindrait 60 ou $70 \%$, par exemple à Nahariya et à Kiryat Shmona. ${ }^{26}$ 
[12b] P1 :Depuis le début de la guerre, plus de 2070 roquettes sont tombées sur le nord d'Israël.

P2 : Selon les estimations en Israël, la moitié des habitants de cette région, soit 800.000 personnes, l'ont fuie pour le centre et le sud du pays.

P3 : Dans les villes qui ont été très gravement touchées,

$\mathrm{P} 4$ : ce chiffre atteindrait 60 ou $70 \%$, par exemple à Nahariya et à Kiryat Shmona.

\section{Construction dynamique du graphe associé à l'exemple [10a] :}

Tout énonciation ouvre un référentiel énonciatif global quant bien même celui-ci n'est pas explicitement marqué dans le texte. On construit ici $\mathrm{RE}_{\mathrm{G}}$, premier nœud du graphe :

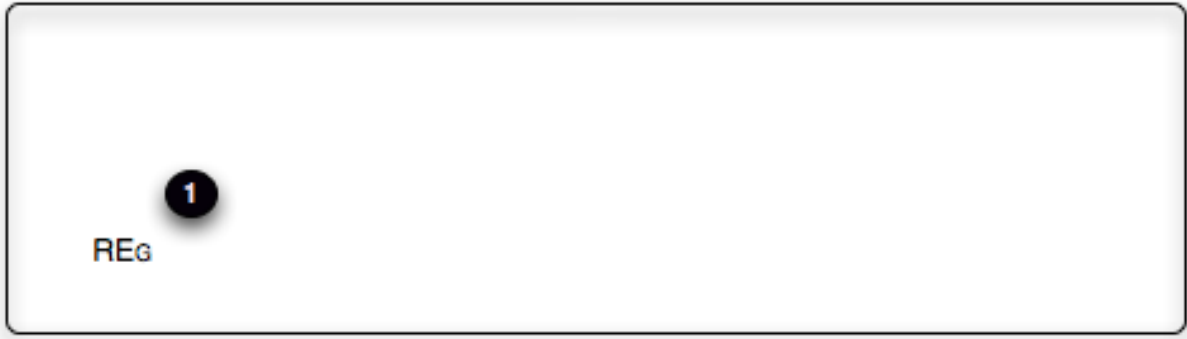

En l'absence d'indice de rupture, P1 est situé sur ce premier référentiel :

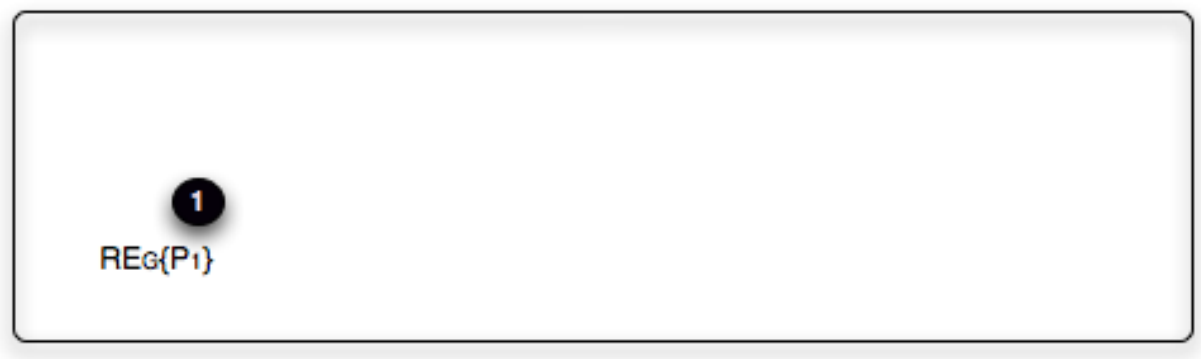

Des indices de ruptures de citation contextualisée («selon» suivi de "estimation») ouvrent un nouveau référentiel $\mathrm{RE}_{\mathrm{L} 1}$ sur lequel est situé $\mathrm{P} 2$ :

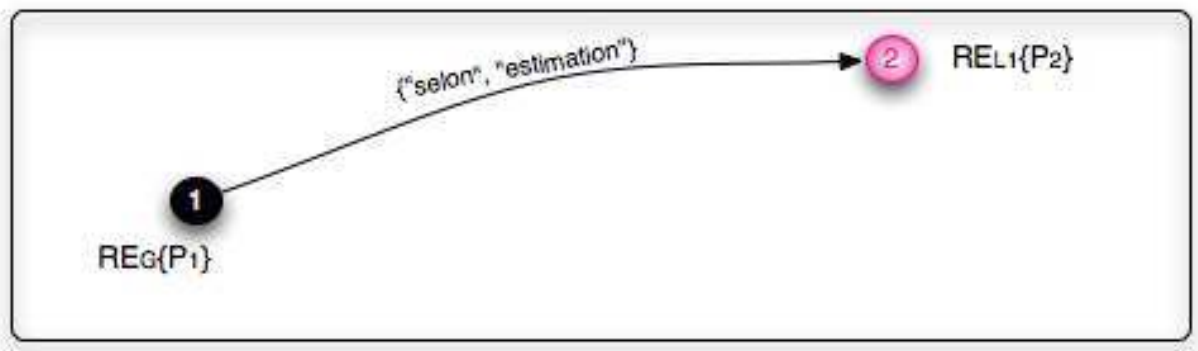

Le mode de « atteindrait » est un indice qui permet d'ouvrir un référentiel $\mathrm{RE}_{\mathrm{E} 1}$ sur lequel se situe P4. Le point final, en tant que marqueur de fin d'énonciation, ferme tous les référentiels ouverts et permet un retour à $\mathrm{RE}_{\mathrm{G}}$ : 


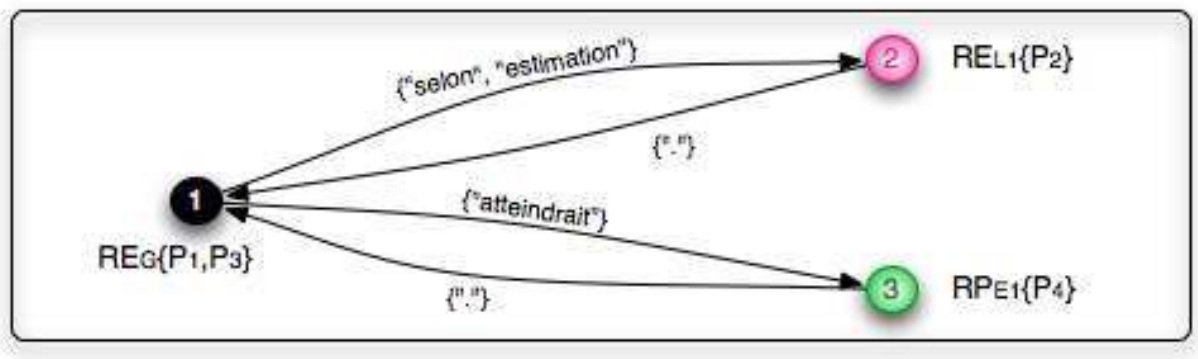

\subsection{Application à un article complet}

La possibilité de matérialiser la structure de textes volumineux dans un espace restreint peut sembler anecdotique. Dans les faits, l'expérience montre que les autres systèmes de représentation - par exemple la représentation en référentiels temporels, en blocs de cadres de discours ou en blocs de la RDST- ne peuvent s'appliquer qu'à des textes courts qui excèdent difficilement quelques lignes ${ }^{27}$. Dès que le texte porte sur plusieurs pages, la représentation devient complexe et difficilement communicable.

Afin de montrer la pertinence de cette représentation, nous proposons le graphe associé à l'article complet présenté dans l'exemple [13]

[13]Hier après-midi, quarante-huit heures après l'ouverture du front libanoisraélien, le Hezbollah qui, selon son chef Hassan Nasrallah, disposerait de plus de 10000 roquettes et missiles pointés sur Israël, avait déjà frappé 165 fois le territoire de l'Etat hébreu. Et ce ne pourrait être qu'un début. Ses cadres affirment qu'ils ont les moyens de procéder à une escalade de grande envergure si Tsahal poursuit ses raids sur le sol libanais. «Haïfa, que nous n'avons pas encore pris pour cible, contrairement à ce que disent les Israéliens, pourrait figurer très prochainement parmi nos objectifs, déclare le député du parti chiite Hussein Haj Hassan. Mais, nous sommes également prêts à étendre nos opérations à une échelle beaucoup plus vaste si les attaques contre nos civils perdurent.» La milice chiite a-t-elle pour autant les moyens d'atteindre Tel-Aviv comme l'a écrit le Jane's Defence Weekly, hebdomadaire britannique spécialisé dans les questions de défense? Les responsables islamistes refusent d'infirmer ou de confirmer. Mais un observateur libanais qui connaît bien le Parti de Dieu soutient qu'il dispose de l'armement nécessaire à des tirs en profondeur. "Le Hezbollah peut aussi se tourner vers un autre plan et décider de frapper à l'extérieur de l'Etat hébreu ", ajoute-t-il. Pour l'heure, les miliciens arrosent le nord d'Israël de leurs roquettes. Disséminés dans le sud du Liban, ils sont lourdement armés. Leur arsenal compterait, entre autres, missiles antichars, mortiers, lance-roquettes $\mathrm{B} 7$, une artillerie antiaérienne ou encore des unités d'artillerie. Un équipement fourni par Téhéran et qui transite par Damas. Depuis le début de son opération, Tsahal a veillé à empêcher tout transfert d'armes vers la frontière libanaise en pilonnant les principaux points de passages vers le sud. Mais l'autre force du Hezbollah, qui a vu le jour après l'invasion israélienne de 1982, réside dans sa capacité de mobilisation de la population. L'immense majorité des Libanais chiites sont acquis à la cause de la résistance islamique et vouent un véritable culte à Nasrallah, élu par ses pairs en 1992. Vendredi, le leader charismatique a échappé de peu au bombardement de son QG dans la banlieue de Beyrouth et promis à la télévision. Alors que le Parti de Dieu a intégré pour la première fois le gouvernement en juillet, il consacrait jusqu'ici l'essentiel de son énergie à la lutte contre l'occupation israélienne. Pour ce faire, il dispose de dizaines de milliers d'hommes en âge de se battre. Parmi eux, 5000 miliciens, dont quelques centaines de commandos seraient particulièrement 
aguerris. Ses objectifs : libérer les fermes de Chebaa, un territoire de $25 \mathrm{~km} 2$ au sud du pays, défendre le pays contre toute agression de Tsahal et obtenir le retour de cinq Libanais détenus en Israël. Des objectifs exclusivement nationaux, donc, tout comme l'est son discours. Malgré tout, ses affinités affichées avec l'Iran, la Syrie ou le Hamas, sèment le doute. "Lors des récentes réunions de la conférence de dialogue national [au Liban, ndlr], Nasrallah a promis aux leaders politiques qu'il veillerait à ce que l'été soit calme, rappelle l'éditorialiste du quotidien AnNahar, Rosana Boumonsef. Or, nous assistons actuellement à une offensive israélienne sans précédent depuis 1982. Alors de deux choses l'une. Soit le Hezbollah a mal calculé les répercussions qu'aurait la capture de deux soldats israéliens. Soit, il a été poussé par l'Iran à venir en aide au Hamas, actuellement considérablement affaibli à Gaza. $»^{28}$

Figure 3 : graphe discursif associé à l'exemple [13]

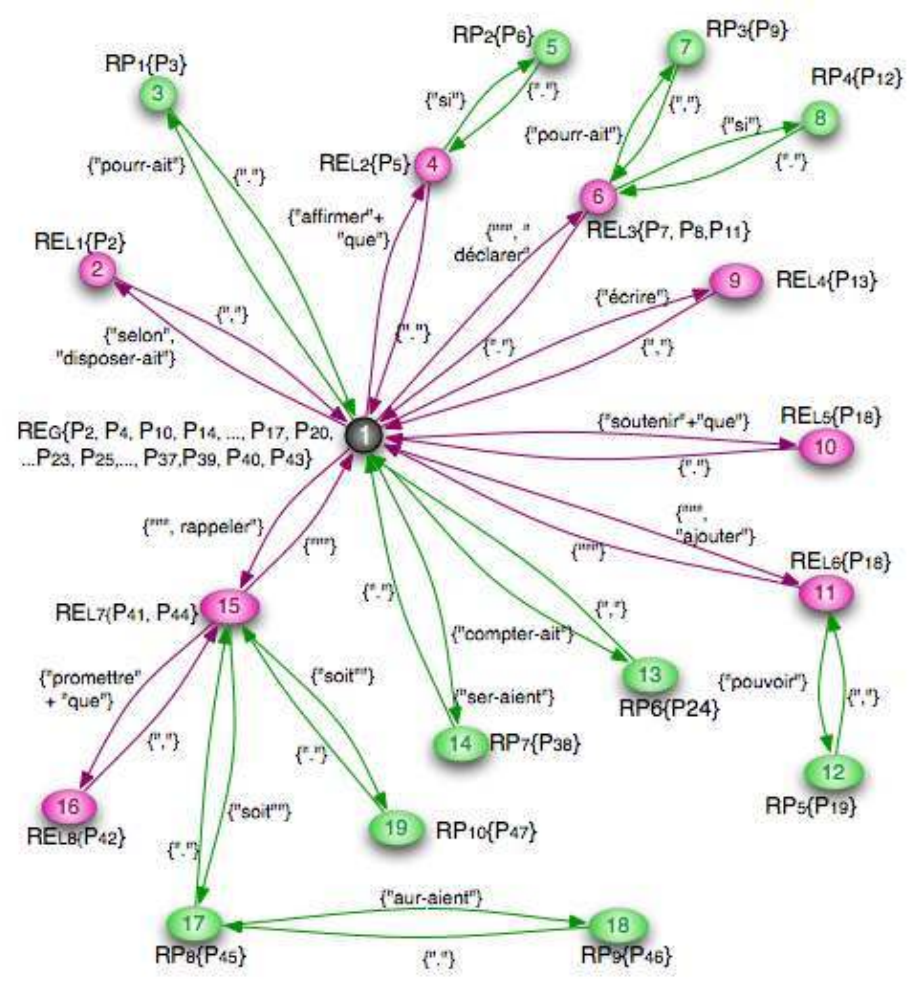

Cette représentation graphique permet de conserver tous les éléments de l'analyse linguistique mais dans un souci de lisibilité, il est possible de supprimer les étiquettes des arcs et de transformer les arcs en arêtes comme dans la figure 3. On peut ensuite ordonner la représentation comme dans la figure 4 pour exploiter la profondeur $\mathrm{du}$ graphe ${ }^{29}$. On accède alors à la structure du texte en tant qu'arbre : n'ayant qu'une seule source et pas de cycle, la structure de données associée au texte est en réalité un arbre au sens mathématique. Il s'agit d'un arbre enraciné dont la racine $r$ correspond au référentiel énonciatif global. Puisque l'ensemble des enfants de chaque noeud est totalement ordonnée, il se définit également comme un arbre orienté. Quand un nouveau référentiel s'ouvre: le noeud est partitionné en ensembles qui sont eux-mêmes des arbres. 
Figure 4 : Arbre discursif

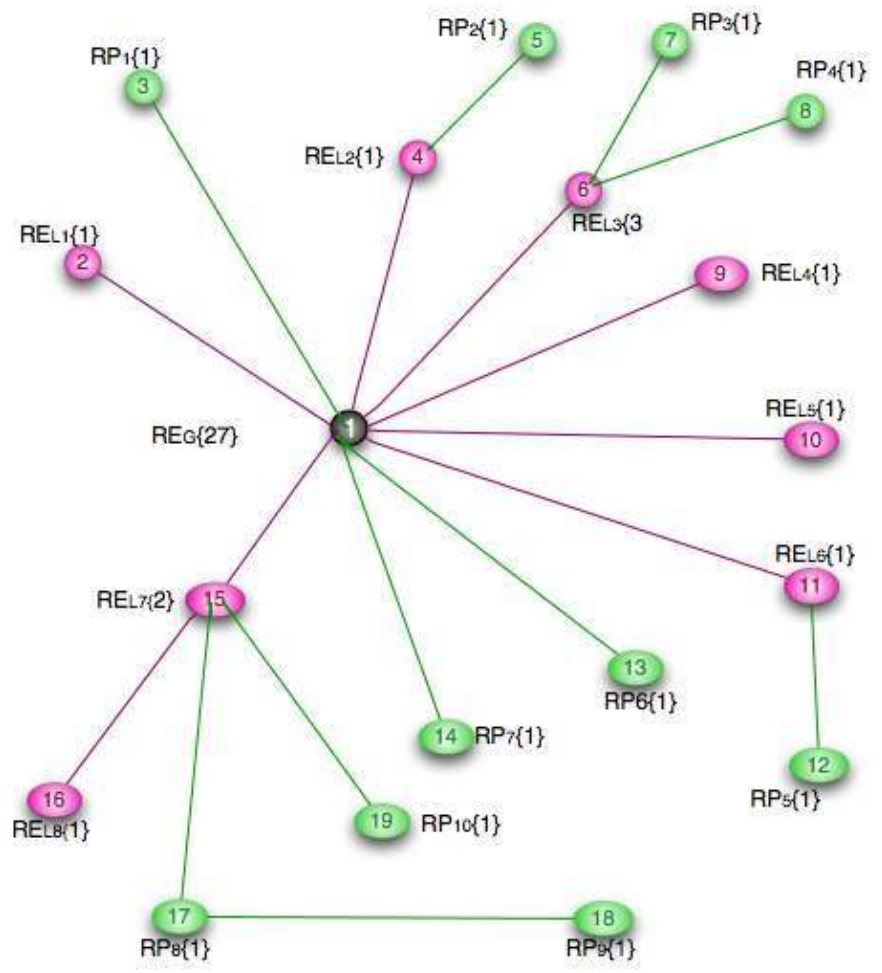

Figure 5 : Arbre discursif ordonné

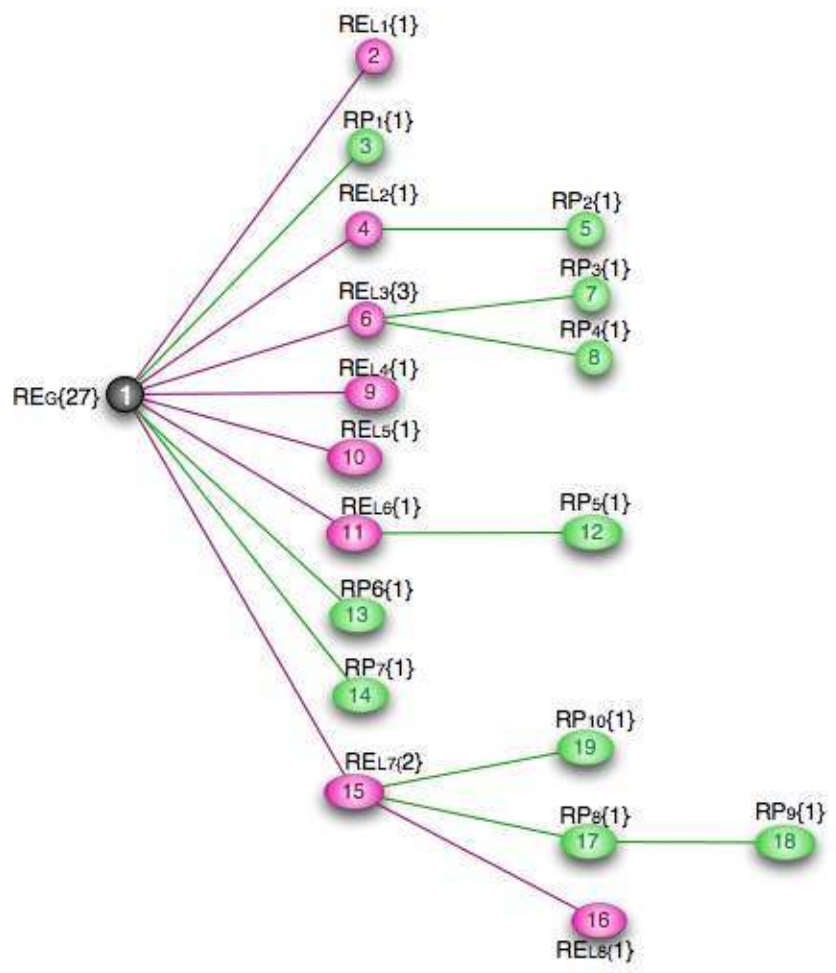

Il est alors possible de définir un arbre textuel par :

- une hauteur $h$ qui correspond à la plus grande profondeur d'une feuille de l'arbre, 
- une taille $t$ qui correspond aux nombres de noeuds en comptant les feuilles,

- une longueur de cheminement $l$ qui correspond à la somme des profondeurs de chacune des feuilles,

- $p$, le nombre de propositions du texte.

\section{Exploitation des résultats}

\section{1 Les avantages de l'analyse}

à la complexité structurelle d'un texte; (ii) l'économie d'un métalangage; (iii) la
possibilité de matérialiser la structure de textes volumineux dans un espace restreint ; (iv) l'exploitation des données de l'arbre.

51 L'accès immédiat à la complexité structurelle d'un texte est l'élément le plus pertinent pour notre étude : il permet de caractériser un texte suivant la complexité de l'arbre qui lui est associé. Exploiter la profondeur de l'arbre ouvre la perspective d'une typologie des articles basés sur des critères énonciatifs et modaux. Il est ainsi possible de fonder intrinsèquement une distinction entre d'une part les articles produits par les autorités israéliennes et palestiniennes et d'autre part les articles de la presse française. Les premiers se caractérisent par une structure simple avec peu de référentiels distincts dont la profondeur n'excède jamais 2 alors que les seconds présentent des emboîtements complexes de référentiels.

Ainsi la figure 6 illustre la structure énonciative d'un texte pro palestinien ${ }^{30}$ : toutes les propositions sont pleinement assumées par l'auteur de l'article. Le référentiel énonciatif global comprend donc les 15 propositions du texte sans qu'aucune rupture énonciative ou modale ne vienne rompre la prise en charge.

Figure 6 : exemple de texte intégralement assumé par l'énonciateur

L'économie du métalangage est rendue possible par la représentation iconique : il suffit de savoir qu'à chaque noeud correspond un type de prise en charge énonciative (un énonciateur ou une modalité) et de distinguer le premier noeud pour savoir si un texte est fortement citationnel ou fortement modalisé.

La possibilité de matérialiser la structure dans un espace restreint permet un affichage graphique qui modifie le rapport au texte puisqu'il est possible de visualiser directement la complexité du texte pour faire émerge d'autres caractéristiques de la structure discursive.

L'exploitation des données de l'arbre associe à chaque texte un ensemble de caractéristiques numériques: nombre de ruptures, nombre et type de référentiels convoqués, etc.

Le tableau 2 illustre quelques-unes des données tirées de la représentation de la figure 5 qui pourront être exploitées par la suite.

Tableau 2 : données numériques associées à l'exemple [1 1]

\begin{tabular}{|l|l|l|}
\hline Type des référentiels & Nombre de propositions & Pourcentage \\
\hline
\end{tabular}




\begin{tabular}{|l|l|l|}
\hline Référentiel énonciatif global & 27 & $56 \%$ \\
\hline Référentiels énonciatifs locaux & 11 & $23 \%$ \\
\hline Référentiels possibles & 10 & $21 \%$ \\
\hline
\end{tabular}

On constate que seulement $56 \%$ des propositions du texte sont pleinement assumées par l'énonciateur contre $100 \%$ dans le cas du texte illustré par la figure 5 .

\section{2 Limites de l'analyse}

Le formalisme de notre approche nous a permis de l'automatiser au sein d'un système de reconnaissance des différents référentiels basé sur des heuristiques linguistiques. L'implantation a été réalisée au sein du système Chronotexte, dont les principes ont été présentés dans [Chagnoux 2006]. Nous ne présentons pas ici les détails de l'automatisation déjà exposés dans [Battistelli \& Chagnoux 2007] mais insistons sur le fait que l'implémentation des règles heuristiques dans un système automatique d'annotation de la structure discursive de ce corpus nous a permis d'évaluer la pertinence de notre approche méthodologique comme la qualité du système implémenté. Par conséquent, les difficultés rencontrées sont de deux ordres : les premières relèvent des choix théoriques, les secondes d'une couverture insuffisante des phénomènes par le système automatique.

\subsubsection{Limites du cadre méthodologique}

59 En choisissant comme unité sémantique de base des propositions construites autour de verbes conjugués, une partie des informations pertinentes échappe parfois à l'analyse. Tout d'abord, il est impossible de rendre compte des phrases nominales comme dans l'exemple [14] où " une information confirmée peu après par une source aéronautique libanaise » ne peut être traduite dans l'arbre.

[14]La télévision du Hezbollah rapporte que l'aviation israélienne a bombardé jeudi matin l'aéroport international de Beyrouth. Une information confirmée peu après par une source aéronautique libanaise..$^{31}$

Ensuite, l'absence de marqueurs explicites au sein du discours indirect entraine souvent une ambiguïté sur la personne qui prend en charge certains termes. Ainsi, dans l'exemple [15], le qualificatif de "sévère » est attribué à l'énonciateur global alors qu'il est plus probable qu'il participe à la citation des propos de l'énonciateur local, Israël.

[15]Israël a aussitôt annoncé une riposte sévère. ${ }^{32}$

61 Enfin, comme l'illustre l'exemple [16a], considérer la proposition comme unité de base fait que des constructions syntaxiques internes à la proposition ne sont pas prises en compte et entraîne une perte d'information. La modification de l'exemple [16a] en [16b] montre que pour un contenu sémantique presque équivalent, seule une analyse de [16b] identifiera les principes de citations.

[16a]Le bilan des blessés est confirmé par le Maguen David Adom, l'équivalent israélien de la Croix-Rouge. ${ }^{33}$

[16b] Maguen David Adom, l'équivalent israélien de la Croix-Rouge, a confirmé que les blessés étaient au nombre de 11 . 


\subsubsection{Limites du système de traitement automatique}

Il est également difficile de repérer automatiquement les reformulations diverses d'une même information. Dans l'exemple [17], d'une part le système ne permet pas d'articuler la première et la seconde proposition - le fait que c'est un énonciateur local, le général Amir Eshel qui assume la probable longue durée de l'opération - et d'autre part il n'est pas possible d'établir une correspondance discursive entre les propos cités directement par le général -. «Cela va durer longtemps » - et les mêmes propos rapportés par l'auteur de l'article - «L'opération au Liban serait de longue durée ».

[17]L'opération au Liban serait de longue durée. C'est ce que déclare le chef d'étatmajor adjoint de l'armée de l'air israélienne, le général Amir Eshel. Il prévient l'opinion internationale et intérieure que: «Il ne faut pas voir cela comme une question de jours. Cela va durer longtemps $»^{34}$

Enfin, le système de règles reste encore à compléter pour pouvoir traiter certains problèmes complexes que ce corpus a fait émerger. À titre d'exemple, nous pouvons citer le cas de la citation partielle comme dans l'exemple [18] : la citation alterne des marques de discours direct comme les guillemets et des marques de citation indirecte. Le phénomène de citation partielle est très présent dans le corpus étudié et devra être modélisé afin que Chronotexte soit apte à le gérer.

[18]Et le Hezbollah de prévenir: "En cas de riposte israélienne", le mouvement n'épargnera "aucun objectif dans la ville de Haïfa et ses environs".

Un autre problème soulevé par le corpus est le cas de l'emboîtement de citations comme dans l'exemple [19]. Le système n'identifie que deux référentiels énonciatifs: le référentiel énonciatif global de l'énonciateur et le référentiel énonciatif local de la radio militaire israélienne. Or, en réalité, trois référentiels devraient être distingués puisqu'à partir du référentiel énonciatif local s'ouvre un second référentiel énonciatif local, celui des responsables de Tsahal. C'est d'ailleurs sur ce référentiel qu'est située la proposition selon laquelle un blocus général est imposé sur le Liban.

[19]La radio militaire israélienne, citant des responsables de Tsahal, annonce qu'un

blocus général (aérien, maritime et terrestre) est imposé sur le Liban.

5 Du point de vue de l'automatisation, le repérage des citations contextualisées apparait très délicat. Si dans des exemples comme [20], les indices lexicaux (« agence de presse ») et typographiques (présence de guillemets) permettent un repérage de citation, les indices sont souvent difficiles à identifier et occasionnent parfois des erreurs d'analyse. Ainsi, dans l'exemple [21], la polysémie de « conception » rend plausible une citation par une autorité iranienne alors que le texte n'en contient pas puisqu'il s'agit d'une technique d'armement et non d'une conception idéologique.

[20] Selon l'agence de presse gouvernementale égyptienne Mena, les deux hommes ont discuté des «moyens de calmer l'escalade et d'empêcher la situation dans les territoires palestiniens et au Liban de se détériorer ${ }^{35}$.

[21]Le Hezbollah utilise différents types de missiles, parmi lesquels des roquettes Katioushas et Fajr 2, ces derniers étant fabriqués en Syrie selon une conception iranienne.

La multiplication de ces phénomènes fait qu'actuellement la tâche de repérage des citations contextualisées est trop bruitée pour être pertinente. Aussi, le repérage automatique des citations contextualisées sans marque explicite de citation comme les guillemets a été exclu de Chronotexte en attendant de pouvoir intégrer des règles de repérage plus fines. 


\section{Conclusion et perspectives}

(1) sa traditionnelle linéarité, elle permet d'imaginer des parcours de navigation qui restent à exploiter. L'une des perspectives que nous souhaiterions également poursuivre pour évaluer la pertinence de notre approche est celle qui permettrait de corroborer ces résultats avec d'autres expressions du caractère polémique des mêmes sujets. En effet, un article peut être sujet à caution par les réactions qu'il suscite auprès des lecteurs. Un premier exemple est celui de Wikipedia, encyclopédie collaborative où chaque article est rédigé par les utilisateurs. La possibilité de suivre les débats entre les différents rédacteurs grâce à l'historique des contributions et des discussions, souvent passionnées, des participants offre alors un outil privilégié pour repérer les articles les plus polémiques : plus les débats sont nombreux et moins le sujet est consensuel. Dans le cas où les désaccords sont trop importants, l'article peut même être soumis à modération. Un second exemple est offert par la possibilité que proposent maintenant les blogs ou certains sites de presse de réagir et commenter chaque article en ligne. Ici encore, certains articles suscitent nombre de réactions de lecteurs alors que d'autres n'entrainent que peu de commentaires. L'examen quantitatif et qualitatif des réactions des lecteurs permettrait de distinguer les articles plus polémiques que d'autres et de comparer l'approche linguistique défendue ici aux approches statistiques comme celles adoptées par [Viegas \& al 2004] et [Nigam \& Hurst 2004].

\section{BIBLIOGRAPHIE}

Asher, N. 1993. Reference to Abstract objects in Discourse. Kluwer Academic Publishers. 
Authier, J. 1981. Paroles tenues à distances. Matérialités discursives. Lille : Presses Universitaires de Lille.

Battistelli, D., Chagnoux and M., Desclés J.-P. 2007. « Référentiels et ordonnancements temporels dans les textes », Information temporelle, procédures et ordre discursif, Cahiers Chronos 18, Amsterdam/New York.

Battistelli, D. and Chagnoux, M. 2007. « Représenter la dynamique énonciative et modale de textes », TALN 2007, Toulouse : 5-8 juin 2007.

Bilhaut, F. 2006. «Introducteurs intra-prédicatifs d'univers de discours et leur détection automatique ». Schedae, prépublication $n^{\circ} 6$, (fascicule 1) 41-50.

Chagnoux, M. 2006. Temporalité et aspectualité dans les textes français. Modélisation sémanticocognitive et traitement informatique. Paris : Thèse de l'Université Paris-Sorbonne.

Charaudeau, P. and Maingueneau, D. 2002. Dictionnaire d'analyse du discours. Paris : Editions du Seuil.

Charolles, M. 1997. «L'encadrement du discours : univers, champs, domaines et espaces ", Cahiers de Recherche Linguistique, 6 : 1-73. Nancy: LANDISCO.

Charolles, M., Le Draoulec, A., Pery-Woodley M.-P. and Sarda, L. 2005, "Temporal and spatial dimensions of discourse organisation", Journal of French Language Studies: 115-130.

Couto, J. 2006. Modélisation des connaissances pour une navigation textuelle assistée. La plate-forme logicielle NaviTexte. Paris : Thèse de l'Université Paris-Sorbonne.

Cornish, F. 2006. « Relations de cohérence en discours : critères de reconnaissance, caractérisation et articulation cohésion-cohérence, Journée d'étude du CRISCO Organisation des textes et cohérence des discours, Université de Caen, texte publié en ligne en 2006

Culioli, A. 1980. « Valeurs aspectuelles et opérations énonciatives : l'aoristique », in Notions d'aspect, J. Davis et R. Martin (eds), 182-193. Paris : Klincksieck.

Desclés, J.-P. 1980. « Construction formelle de la catégorie grammaticale du temps et de l'aspect », in Notions d'aspect, J. Davis et R. Martin (eds), 198-237. Paris : Klincksieck.

Desclés, J.-P. 1995. Les référentiels temporels pour le temps linguistique. Modèles linguistiques 16 : 9-36.

Desclés, J.-P., and Guentchéva, Z. 2000. « Enonciateur, locuteur, médiateur. » Erikson Ph et Monod-Becquelin A (éds). Les rituels du dialogue : 79-112. Nanterre : Société d'éthnologie. Paris X. Fauconnier, G. 1985. Mental spaces : Aspects of meaning construction in natural language. Cambridge : MIT Press.

Espuny, J. 1998. « De l'énonciation singulière à l'énonciation plurielle du locuteur », Estudios de lengua y literatura francesas, $12: 53-70$, Cadix.

Gosselin, L. 2005. Temporalité et modalité. Bruxelles : De Boeck Duculot

Guentcheva, Z. (éd.) 2000. L'énonciation médiatisée. Louvain-Paris : Peeters.

Hobbs, J.R. 1990. "Chap. 5: The coherence and structure of discourse". Literature and Cognition, Leland Standford Junior University, Calif : CSLI Lecture Notes 21: 83-114.

Kronning, H. 2003. « Modalité et évidentialité ». Birkelund, M., Boysen, G. \& Kjærsgaard, P. S. (éds). Aspects de la Modalite. Tübingen : Max Niemeyer, Linguistische Arbeiten 469 : 131-151.

Leeman-Bouix, D. 1994 Grammaire du verbe français. Fernand Nathan. 
Le Querler, N. 1996. Typologie des modalités, Caen : Presses Universitaires de Caen.

Maingueneau, D. 1994. L'Enonciation en linguistique française. Paris : Hachette.

Martin, R. 2005. « Définir la modalité », Revue de linguistique romane 273 : 7-18. Strasbourg.

Mann, W.C., Thompson, S.A. 1988. "Rhetorical Structure Theory : toward a functional theory of text organization", Text 8(3), 243-281.

Nigam, K. and Hurst, M. 2004. "Towards a robust metric of opinion. In Proc. AAAI Symp. Exploring Attitude and Affect in Text.

Péry-Woodley, M.-P. 2001. "Présentation du numéro : Cohérence et relations de discours à l'écrit », Verbum, XXIII, 1.

Porhiel, S. 2001, "Linguistic expressions as a tool to extract thematic information", Corpus Linguistic, Lancaster: 477-482.

Rabatel, A. 1998. La Construction textuelle du point de vue. Lausanne, Paris : Delachaux et Niestlé.

Rabatel, A. 2006. « L'effacement de la figure de l'auteur dans la construction événementielle d'un « journal » de campagne électorale et la question de la responsabilité, en l'absence de récit primaire Semen, 22.

Recanati, F. 2000. Oratio Obliqua, Oratio Recta: An Essay on Metarepresentation. Cambrige : MIT Press.

Rosier, L. 1993. « De la stylistique sociologique suivie d'une application pratique : discours direct, presse et objectivité », Revue belge de philosophie et d'histoire 71. 625-644.

Rosier, L. 1999, Le discours rapporté. Histoire, théories, pratiques, Bruxelles, Paris : Duculot.

Roulet, E. 1999, La description de l'organisation du discours, Paris, Didier.

Sandor, A., Kaplan, A. and Rondeau, G. 2006. "Discourse and citation analysis with concept matching". Schedae, prépublication $\mathrm{n}^{\circ} 19$, (fascicule 1) 147-152.

Sauri, R., Verhagen, M. and Pustejovsky, J. 2005. Annotating and recognizing Event Modality in Text. Actes FLAIRS'06, Melbourne Beach, Florida.

Schrepfer-Andre, G. 2006. La portée phrastique et textuelle des expressions introductrices de cadres énonciatifs : Les syntagmes prépostionnels en selon X. Paris : Thèse de l'Universtié Paris III Sorbonne Nouvelle.

Terran, E. 2002. Le cadrage temporel en français. Thèse de doctorat, Université Paris III - Sorbonne Nouvelle.

Tuomarla, U. 2000. La citation mode d'emploi. Sur le fonctionnement discursif du discours rapporté direct. Helsinki: Academia scientiarum Fennica.

Viegas, F. B., Wattenberg, M. and Dave, K. 2004. "Studying cooperation and conflict between authors with history flow visualizations". In Proc. SIGCHI Conf. Human factors in computing systems: 575-582.

Vinzerich, A. and Desclés J.-P. 2006. « Référentiels des possibles : Représentation des situations potentielles et irréelles. ». Actes Chronos 7. Anvers, 18-20 septembre 2006.

Wonsever, D. 2004. Repérage automatique des propositions par exploration contextuelle. Paris : Thèse de l'Université Paris-Sorbonne. 


\section{ANNEXES}

\section{Texte associé à la figure 5.}

Source : http://www.populationdata.net/cartes/actus/liban-attaques-18juillet06.php

Israël a unilatéralement décidé d'écraser le Liban sous les bombes, réduisant les efforts de reconstruction des 10 dernières années. Les ponts, aéroports, ports, routes, transports en commun, usines électriques, stations services, postes d'essence, dépôts de nourriture etc... sont détruits méthodiquement, bloquant le pays tout entier. La situation humanitaire devient catastrophique !

Les civils sont particulièrement ciblés, Israël cherchant ainsi à démoraliser et punir toute une population dont une portion qui soutient le Hezbollah. On dénombre au 18 juillet 500000 déplacés (selon l'UNICEF), 216 civils morts et des centaines de blessés. Parmis les morts, environ 30 étrangers dont 7 ou 8 canadiens d'une même famille de Montréal.

Le bilan reste incertain, tant l'accès au Sud Liban reste impossible, ainsi que dans certaines zones du sud de Beyrouth, fief du Hezbollah.

Le bilan des morts en Israël était de 23 morts dimanche 16 juillet.

Les réactions occidentales, outre l'évacuation des ressortissants étrangers, sont tièdes, face aux déclarations des États-Unis qui soutiennent ouvertement Israël et empêchent également toute résolution Onusienne contre cette agression brutale et sans limites.

\section{NOTES}

1. http://fr.wikipedia.org

2. Bien que nous opposions indicatif et conditionnel dans cet exemple, à l'instar, de [D. LeemanBouix 1994], nous pensons qu'il est possible de considérer le conditionnel comme un temps de l'indicatif.

3. Site de l'Express, http://www.lexpress.fr, le dimanche 16 septembre 2007, mis à jour à $14: 17$.

4. Site de l'Express, http://www.lexpress.fr, le lundi 17 septembre 2007, mis à jour à $14: 34$.

5. Libération, 1 juillet 2006.

6. Le Monde, 10 juillet 2006.

7. «L'armée israélienne pénètre sur le territoire libanais, les avions survolent Beyrouth.», http://www.france-palestine.org/article4161.html

8. «L'offensive du Hezbollah - Chronologie des événements. », Site de aul'ambassade d'Israël en France, http://paris1.mfa.gov.il/mfm/web/main/missionhome.asp?MissionID=31\&

9. [Maingueneau 1994 :142].

10. Nous considérons ici que le locuteur est énonciateur puisqu'il se trouve «à l'intérieur de l'acte d'énonciation ». [Charaudeau et Maingueneau $2002: 350]$.

11. [ibid : 189].

12. Les mises en cause de la distinction classique entre les divers types de discours rapporté résumées par [Charaudeau \& Maingueneau 2002 :192-195] nous incitent à aborder les exemples 4 davantage en fonction des marqueurs linguistiques qu'en fonction de la typologie "discours rapporté direct, discours rapporté indirect et discours rapporté indirect libre ».

13. Dans la typologie de [Le Querler 1996], ces modalités sont plus précisément des modalités subjectives épistémiques. 
14. Nous utilisons ici le terme de référentiel tel qu'il est utilisé en physique : en physique, un référentiel est un «système de coordonnées, trois spatiales et une temporelle, auxquelles sont rapportées les équations d'un problème de physique " (TLFi, Trésor de la Langue Française informatisé, http://atilf.atilf.fr/). Un même évènement est donc différent selon l'emplacement où se trouve cet observateur et selon les repères spatio-temporels qu'il a.

15. «L'armée israélienne investit le Liban sud», http://www.rfi.fr/actufr/articles/079/ article_45021.asp

16. [ibid :30].

17. Dans [Battistelli \& al, 2006], nous avions proposé une modélisation des référentiels temporels. Nous reprenons et explicitons ici le même formalisme mais appliqué au traitement de la prise en charge énonciative.

18. Que cette méthodologie soit applicable à tout type de textes ne signifie aucunement qu'elle est pertinente pour tout type de texte : certains textes présentent davantage de variations de prise en charge que d'autres, la méthodologie vise justement à établir une typologie en ce sens.

19. L'objectif de cet article est de décrire les mécanismes de construction de la structure référentielle, par conséquent, nous ne précisons pas ici les analyses détaillées sur lesquelles se fondent cette typologie. Pour le référentiel énonciatif, on pourra consulter [Guentchéva 1996], [Desclés et Guentchéva 2000] et [Chagnoux 2006] et pour le référentiel des possibles, [Vinzerich et Desclés 2007] et [Kronning 2003].

20. Extraits tirés de «Israël frappe lourdement le Sud-Liban », Le Figaro, http://www.lefigaro.fr , édition en ligne du 15/10/07.

21. ibid.

22. «Conflit israélo-libanais de 2006. », http://fr.wikipedia.org/ .

23. «L'offensive du Hezbollah - Chronologie des événements.», http://paris1.mfa.gov.il/mfm/ web/main/missionhome.asp?MissionID=31\&

24. «Israël en guerre contre le Hezbollah.», http://www.liberation.fr/actualite/ monde/193193.FR.php

25. «Conflit israélo-libanais de 2006. », http://fr.wikipedia.org/

26. "L'offensive du Hezbollah - Chronologie des événements. ", http://paris1.mfa.gov.il/mfm/ web/main/missionhome.asp?MissionID=31\&

27. Certaines représentations d'un même texte dans des systèmes différents sont disponibles dans [Chagnoux 2006].

28. «Israël en guerre contre le Hezbollah.», http://www.liberation.fr/actualite/ monde/193578.FR.php .

29. Les figures 3,4 et 5 ne sont que trois représentations différentes d'une même analyse. Le graphe de la figure 3 permet de vérifier la pertinence de l'analyse linguistique alors que l'arbre de la figure 5 servira davantage à illustrer la complexité de la dynamique énonciative.

30. L'intégralité du texte est donné en annexe.

31. "Israël en guerre contre le Hezbollah.», http://www.liberation.fr/actualite/ monde/193193.FR.php.

32. «Israël frappe lourdement le Sud-Liban. », Le Figaro, http://www.lefigaro.fr/ , édition en ligne du 15/10/07.

33. «Israël en guerre contre le Hezbollah», http://www.liberation.fr/actualite/ monde/193193.FR.php.

34. Ibid.

35. «L'armée israélienne investit le Liban sud », Arsenault, C., http://www.rfi.fr/ 


\section{RÉSUMÉS}

Les phénomènes de citation et de modalisation du discours permettent aux auteurs de sujets d'actualité d'informer sans s'engager personnellement sur la véracité de leurs propos, voire même de faire figurer dans un même article des informations contradictoires. Nous proposons dans cet article un cadre méthodologique pour repérer et représenter la dynamique textuelle liée à ces variations de prise en charge énonciative sous forme d'arbre discursif. La profondeur de l'arbre associé à un texte témoigne alors de la complexité des différents référentiels en jeu. L'objectif de cette approche, directement implémentable, est de faire émerger automatiquement d'un corpus les textes sujets à controverses.

Through discourse attribution and modality mechanisms, newspeople can convey information without having to personally endorse what they are reporting. In some cases, they may even combine news from conflicting sources. We therefore provide a methodological framework aiming at detecting, locating and representing commitment as well as modality dynamics in texts, using discursive trees. In particular, the depth of a tree associated to a text illustrates the complexity of its reference frames. The purpose of this approach, more broadly, is to automatically exhibit contentious texts from any given corpus.

\section{INDEX}

Mots-clés : représentation discursive, énonciation, citation, arbres discursifs

Keywords : discursive model, commitment, attribution, discursive trees

\section{AUTEUR}

\section{MARIE CHAGNOUX}

MALIRE - LIP6, Université Pierre et Marie Curie, marie.chagnoux@lip6.fr 